\title{
Continuous costoclavicular brachial plexus block in a pediatric patient for postfracture rehabilitation
}

\author{
Rita Regufe $^{1}$ (D) | Vanessa Artilheiro ${ }^{2}$ | Maria Beatriz Dias ${ }^{3}$ | Ivanete Miranda ${ }^{4}$ | \\ Teresa Cenicante ${ }^{4}$
}

\author{
${ }^{1}$ Anesthesiology Department, Centro \\ Hospitalar de Setúbal, Setúbal, Portugal \\ ${ }^{2}$ Anesthesiology Department, Centro \\ Hospitalar Lisboa Ocidental, Lisbon, \\ Portugal \\ ${ }^{3}$ Anesthesiology Department, Hospital da \\ Luz, Lisbon, Portugal \\ ${ }^{4}$ Anesthesiology Department, Hospital Dona \\ Estefânia, Centro Hospitalar Lisboa Central, \\ Lisbon, Portugal \\ Correspondence \\ Rita Regufe, Anesthesiology Department, \\ Centro Hospitalar de Setúbal, Rua Camilo \\ Castelo Branco, 2910-446 Setúbal, Portugal. \\ Email: rfregufe@hotmail.com
}

Editor: David Polaner

\begin{abstract}
The costoclavicular approach to the brachial plexus block has been recently described as a technique for anesthesia or postoperative analgesia of distal upper limb. In this article, we describe a case in which a continuous costoclavicular brachial plexus block was performed in a pediatric patient for conservative treatment of a traumatic radial fracture with severe elbow rigidity. Perineural catheter placement is a valuable option for pain control and functional prognosis during rehabilitation.
\end{abstract}

\section{KEYWORDS}

analgesia, brachial plexus block, pain management, pediatrics, perineural catheter, rehabilitation
The costoclavicular approach to the brachial plexus block has been recently described as a relatively easy-to-perform technique with rapid block onset, with similar distribution to an infraclavicular approach. ${ }^{1}$ For anesthesia or analgesia, this block has been used with good results for elbow and lower arm surgery. ${ }^{2}$ We describe a successful continuous ultrasound-guided costoclavicular brachial plexus block for physical rehabilitation in a pediatric patient.

A twelve-year-old girl suffered traumatic dislocation of left elbow with aligned radial head fracture and underwent conservative treatment with cast immobilization for one month.

After cast removal, severe articular rigidity was observed with extension limited to 45 degrees and flexion limitation at 60 degrees. This restraint was accompanied by severe joint pain, sustaining resting pain Visual Analogue Scale (VAS) scores of 2 and 8 with active or passive movements, respectively.

A computer tomography scan revealed an avulsion fracture of the coronoid process. Conservative treatment with physical rehabilitation was planned, as a surgical approach would increase articular rigidity. Engagement to rehabilitation workout was severely compromised by relentless pain. The patient was proposed for placement of a brachial plexus perineural catheter.

After induction of general anesthesia, the left arm was abducted to 90 degrees and patient's head slightly turned right. Under aseptic technique, a high-frequency linear ultrasound transducer was placed over infraclavicular fossa and tilted caudally until visualization of the brachial plexus' three cords, external to axillary artery. A $18 \mathrm{G}$ perineural catheter Contiplex ${ }^{\circledR}$ Tuohy Ultra $360^{\circ}$ (B-Braun), with $50 \mathrm{~mm}$ needle, was used. The needle was advanced in-plane, medial to lateral. After injection of $5 \mathrm{~mL}$ ropivacaine $0.2 \%$, the catheter was placed near the perineural sheath. Catheter tip position was confirmed through ultrasound visualization of the adequate dispersion of $1 \mathrm{~mL}$ of the same solution. The catheter was fixed to skin with surgical glue.

Recovery at the Post-Anesthetic Care Unit provided initial evaluation of peripheral nerve block efficacy, namely painless mobilization, hypoesthesia of forearm and improved stiffness.

The catheter remained fixed for two weeks, with its filter substituted after one week. Seven physiotherapy treatments were done under regional analgesia and IV acetaminophen. About $5 \mathrm{~mL}$ of ropivacaine $0.2 \%$ was administered 20 minutes before each session. Once the block was installed, physiotherapy was initiated. Treatments lasted one hour and consisted of a series of passive, active, and against resistance movements. The exercises involved a combination of activities regarding elbow flexion/extension; arm internal/external rotation; shoulder abduction/adduction; wrist flexion/extension/lateral deviation; and hand opening/closing. Each 
session was completed with massage and acupressure of trigger points, at elbow and wrist muscular insertions.

The analgesic block lasted nearly two hours, with effective pain control during physiotherapy-maximum VAS 3-and slight motor block without interference in her performance. After five treatments, a sustained progressive gain was observed in elbow range motion, with flexion reaching 120 degrees (60 degrees over initial limitation) and extension to a remainder of 20 degrees (improvement of 25 degrees from pretreatment values). Oral acetaminophen, without perineural bolus, was administered before the eighth physiotherapy session, with a maximum VAS score of 4 , allowing a smooth transition in pain management.

The perineural catheter was removed after fifteen days. No events were recorded. The patient was discharged to maintain the rehabilitation process in an outpatient setting.

Brachial plexus blocks are usually implemented for upper limb surgery or postoperative pain. Recently, the costoclavicular approach has been described as an easy-to-perform technique. At this level, with correct patient positioning, the cords stay clustered together, facilitating brachial plexus identification and correct local anesthetic injection. ${ }^{1}$ Its applicability in postfracture rehabilitation is not described in the literature.

A continuous costoclavicular brachial plexus block seemed the best choice to reduce the probability of catheter displacement. ${ }^{3}$ We decided on a medial to lateral approach to leave the catheter away from the shoulder, preventing accidental exteriorization. The pattern of analgesia and paresthesia remained consistent during all ropivacaine boluses, excluding catheter displacements.

Ropivacaine boluses provided some muscle relaxation, facilitating patient's performance during physiotherapy, and contributed to improve elbow range motion.

There was no need for opioid analgesia. Both patient and mother revealed high satisfaction levels regarding this regional technique.

No infectious or inflammatory complications were observed. Careful manipulation of the catheter, aseptic technique placement, and close monitoring suppressed the need for antibiotic prophylaxis.

\section{1 | LEARNING POINTS}

1. Prolonged use of peripheral nerve catheters can facilitate rehabilitation when conservative measures have failed.

2. The insertion site of the peripheral nerve catheter using the costoclavicular brachial plexus approach is convenient when long dwell time is anticipated.

3. Intermittent bolusing prior to physical therapy can provide adequate analgesia and enhance rehabilitation.

\section{DISCLOSURES}

The authors report no conflict of interest.

\section{ETHICS APPROVAL}

Informed consent was obtained for publication.

\section{ORCID}

Rita Regufe (iD https://orcid.org/0000-0001-7276-6820

\section{REFERENCES}

1. Li J, Songthamwat B, Samy W, Sala-Blanch X, Karmakar MK. Ultrasound-guided costoclavicular brachial plexus block. Reg Anesth Pain Med. 2017;42(2):233-240.

2. Leurcharusmee P, Elgueta MF, Tiyaprasertkul W, et al. A randomized comparison between costoclavicular and paracoracoid ultrasound-guided infraclavicular block for upper limb surgery. Can J Anesth. 2017;64:617-625.

3. García-Vitoria C, Vizuete J, López Navarro AM, Bosch M. Costoclavicular space, a reliable gate for continuous regional anestesia cateter insertion. Anesthesiology. 2017;V127(4):712.

How to cite this article: Regufe R, Artilheiro V, Dias MB, Miranda I, Cenicante T. Continuous costoclavicular brachial plexus block in a pediatric patient for postfracture rehabilitation. Pediatr Anesth. 2020;30:720-721. https://doi. org/10.1111/pan.13875 